\title{
Composition and Functional Diversity of the Urban Flora of Alfenas-MG, Brazil
}

\author{
Nathalia Monalisa-Francisco ${ }^{1}$ (D), Flavio Nunes Ramos ${ }^{1}$ \\ ${ }^{1}$ Programa de Pós-graduação em Ciências Ambientais, Instituto de Ciências da Natureza, Universidade Federal de Alfenas \\ - UNIFAL, Alfenas/MG, Brasil
}

\begin{abstract}
Urban tree cover has important environmental and social functions and can act as ecological refuges. The objective of the present study was to investigate the taxonomic and functional diversities of urban plant communities in Alfenas, Minas Gerais State, Brazil. We sampled all trees $\mathrm{DBH} \geq 3 \mathrm{~cm}$ in eight different urban green areas, recording 1,138 individuals and 119 species; two species were dominant: Poincianella pluviosa (Fabaceae) and Syagrus romanzoffiana (Arecaceae). The high species richness encountered reflected, in part, the presence of exotic species, which corresponded to $40 \%$ of the species and $25 \%$ of the total abundance. The functional diversity index (HF') was considered low, with the predominant functional traits among the species being small size, entomophily, zoochory, evergreen leaves, and dry fruits. We recommend that future urban afforestation projects incorporate strategies that increase the use of regional species as well as the functional diversity/complexity of those environments.
\end{abstract}

Keywords: functional diversity, green areas, regional species, urban ecology, urban trees. 


\section{INTRODUCTION}

The global human population has increased approximately ten-fold in the last century. Urban areas currently occupy ca. $4 \%$ of the total earth surface (Töpfer et al., 2000), which considerably increases the importance of conserving biological communities in urban ecosystems. Green urban areas have significant potential to aid biodiversity conservation, provide diverse advantages to human populations (see Roy et al., 2012), contribute to the environmental quality of cities, and act as refuges for rich plant communities (Ordóñez \& Duinker, 2012; Freitas et al., 2015).

Despite their potential as biodiversity refuges, urban green spaces tend to have quite peculiar floristic compositions. The presence of exotic species is a key factor determining diversity patterns in those ecosystems. They represent a substantial component of urban forests not only in Brazil but around the world (Aronson et al., 2007; Bigirimana et al., 2011; Kowarik, 2011; Kramer \& Krupek, 2012). The ecological roles of exotic species remain controversial. Some evidence point to their undesirable effects on local plant communities (McKinney, 2006), while other evidence suggest their positive performance within the environmental balance of urban areas (Schlaepfer et al., 2011). There is no consensus on the use of exotic plants in urban afforestation, at the same time that the silvicultural potentials of regional native species have largely been neglected (Isernhagen et al., 2009).

Although studies of diversity patterns based on composition and natural occurrence are quite useful, functional diversity has been shown to be a promising approach for understanding ecological issues in anthropogenic habitats (Cornelissen et al., 2003; Duncan et al., 2011). The characteristics of a species, rather than its identity, will determine its contribution to ecosystem functioning (Díaz \& Cabido, 2001; Knapp et al., 2010). The quantification and understanding of plant community characteristics and functions will allow more appropriate conservation and management decisions. Some researchers have investigated plant ecological attributes in urban ecosystems. They described phenomena such as the directional selection of specific traits (Aronson et al., 2007; Duncan et al., 2011) and functional redundancy along richness gradients (Knapp et al., 2008). Most studies on urban afforestation, however, have focused principally on taxonomic diversity (Cardoso-Leite et al., 2014; Freitas et al., 2015; Kramer \& Krupek, 2012), with little emphasis on the functional diversity of those plant communities.

Since tropical forest physiognomies have been increasingly converted into anthropically altered environments, conservation strategies are urgently needed. They can help transform urban ecosystems into ecological refuges - and those transformations will require basic information about community functioning in those environments. The objective of this study was to investigate ecological patterns of plant communities in urban public green areas and describe aspects of their floristic compositions and functional and taxonomic diversities.

\section{MATERIALS AND METHODS}

\subsection{Study area and sampling}

The present study was conducted in Alfenas $\left(21^{\circ} 25^{\prime} 46^{\prime \prime}\right.$ S; $\left.45^{\circ} 56^{\prime} 50^{\prime \prime} \mathrm{W}\right)$, Minas Gerais State, Brazil. The municipality covers $850 \mathrm{~km}^{2}$ and has approximately 74,000 inhabitants. The natural vegetation is Semideciduous Seasonal Atlantic Forest (Ibge, 2012), with dry winters and wet summers (Cwa type climate, Köppen 1948). The mean regional temperature ranges from $17.5^{\circ} \mathrm{C}$ during the Austral winter to $21.1{ }^{\circ} \mathrm{C}$ in the summer, with a mean annual precipitation of $1500 \mathrm{~mm}$ (Alvares et al., 2014). Eight public green areas were chosen for study. Most of them lie within the urban perimeter of the municipality, except the Alfenas Municipal Park, located a few kilometers from the city (Figure 1).

Those areas represent a significant percentage of the cultivated vegetation cover in the city, are easily accessed by the local population, and are commonly used for social activities. Each of those green areas was visited between 2011 and 2012. All trees with $\mathrm{DAP} \geq 3 \mathrm{~cm}$ were mapped, photographed, and identified with the help of specialized bibliography and/or expert consultations.

\subsection{Species classifications}

The species were classified - using information available in the specialized scientific literature and/or expert consultations - according to their geographical origins and ecological characteristics. 

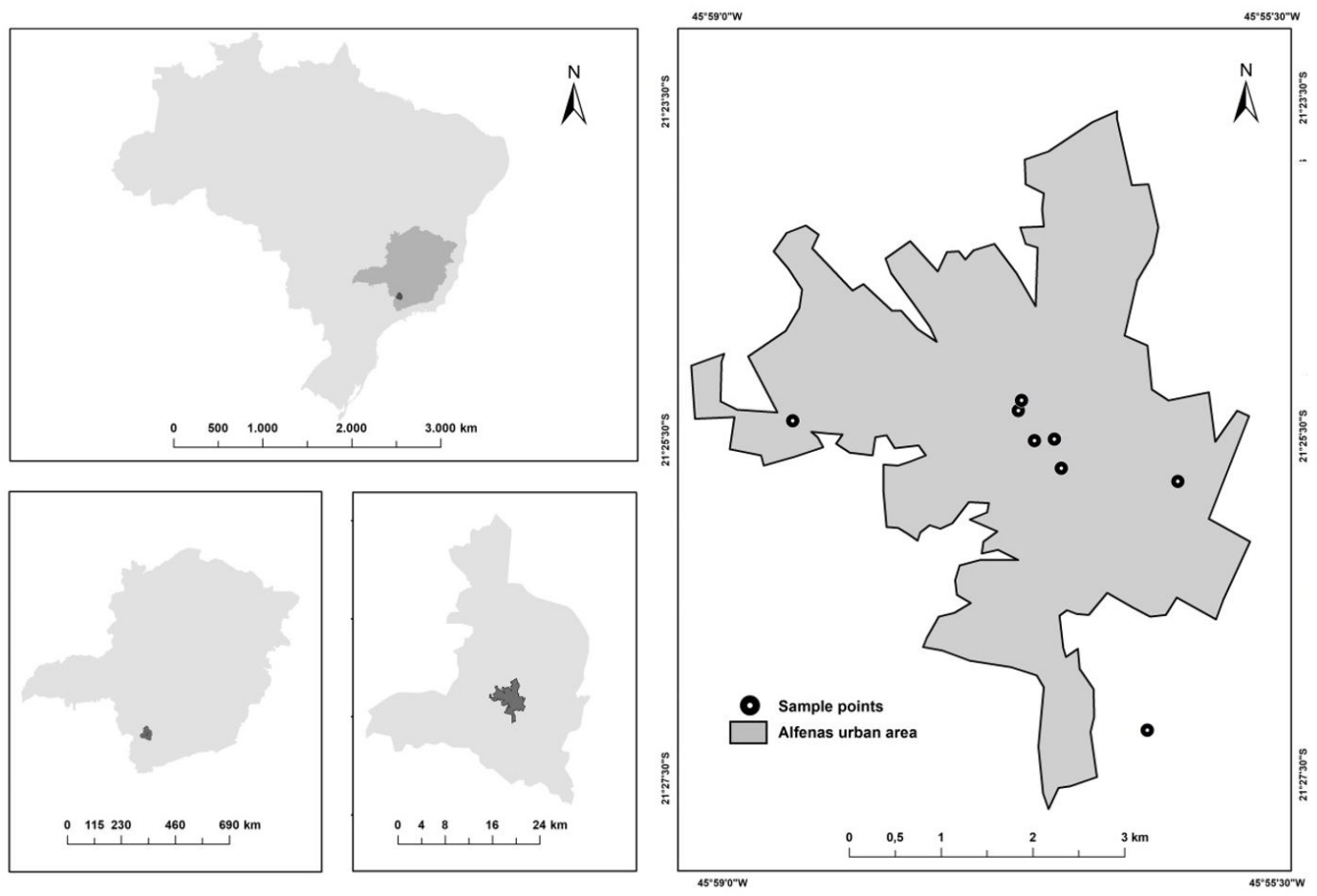

Figure 1. Location of the eight green public areas studied in Alfenas, Minas Gerais State, Brazil.

(1) Geographical origin: regional native species occur naturally in the same phytophysiognomy of the study area (Semideciduous Seasonal Atlantic Forest); native species are those occurring in other Brazilian formations; and exotic species were those having no recorded natural occurrence in any Brazilian vegetation.

(2) Ecological attributes: were chosen the ecological attributes that represent important characteristics for the performance of ecosystem functions and contribution to environmental balance in cities, adapted from Cornelissen et al. (2003) and Duncan et al. (2011):

a) Tree size: small $(\leq 12 \mathrm{~m})$; medium $(12-20 \mathrm{~m})$; and tall $(>20 \mathrm{~m})$.

b) Pollination mode: anemophilous, ornithophilous, entomophilous, and chiropterophilous.

c) Dispersal mode: autochoric, hydrochoric, anemochoric, and zoochoric.

d) Leaf life span: deciduous or evergreen leaves.

e) Fruit type: fleshy or dry fruits.

\subsection{Statistical analysis}

The general patterns of species diversity were described based on abundance, richness, and evenness. Evenness was calculated using the Pielou J' index, whose values range from 0 to 1 reflecting the distribution uniformity of individuals within the species. The higher the J' value, the more balanced is the community composition.

To describe community structures, we also investigated dominance patterns, classifying the species according to their total abundance as: a) rare (those with only 1 individual); b) few abundant (2-5 individuals); c) abundant (6-50 individuals); d) intermediate (51-100 individuals); and e) dominant (> 100 individuals).

To investigate the functional diversity patterns (DivF) we employed a functional diversity index adapted from Shannon \& Weaver ( $\left.H^{\prime}\right)$ using the relative proportion of individuals (HF'ind) and species (HF'sp) within each group: $H F^{\prime}=-\sum p_{i}^{*} \operatorname{Ln}\left(p_{i}\right)$, where $p_{i}=$ the abundance or richness of the $i$-category.

We used analyses of variance to verify whether the mean proportions of individuals and species (after arcsine square root transformation) differed between 
ecological categories. The nonparametric equivalent tests (Mann-Whitney, Kruskal-Wallis) were used with data that could not be corrected by transformations in terms of the normality and homogeneity of variances. The level of significance considered was $5 \%$.

\section{RESULTS}

We surveyed 1,138 individuals belonging to 119 species, 101 genera, and 43 families. The most representative family was Fabaceae (19 species and
290 individuals). The five most abundant species were Poincianella pluviosa var. Peltophoroides (Benth.) L.P. Queiroz (237 ind), Syagrus romanzoffiana (Cham.) Glassman (122 ind), Handroanthus impetiginosus (Martius ex. DC.) Mattos (72 ind), Ficus benjamina L. (59 ind), and Pleroma granulosum (Desr.) D. Don (55 ind). Together these species accounted for $48 \%$ of the total abundance (Table 1).

In terms of geographic groups, there was a notable presence of exotic species $(40 \%)$ in relation to native (23\%) and regional species $(37 \%)$. Concerning to

Table 1. Information about the urban flora in Alfenas, Minas Gerais State, Brazil.

\begin{tabular}{|c|c|c|c|c|c|c|c|c|}
\hline Family & Species & Total & Ori & Size & Pol & Dis & Leaf & Fru \\
\hline \multirow{5}{*}{ ANACARDIACEAE } & Lithraea molleoides (Vell.) Engl. & 2 & $\mathrm{~N}$ & Sma & Ent & Zoo & Eve & Dry \\
\hline & Mangifera indica L. & 11 & $\mathrm{E}$ & Tall & Ent & Zoo & Eve & Fle \\
\hline & Schinus molle L. & 53 & $\mathrm{~N}$ & Sma & Ent & Zoo & Eve & Fle \\
\hline & Schinus terebinthifolia Raddi & 1 & $\mathrm{R}$ & Sma & Ent & Zoo & Eve & Fle \\
\hline & Tapirira guianensis Aubl. & 1 & $\mathrm{R}$ & Sma & Ent & Zoo & Eve & Fle \\
\hline ANONNACEAE & Annona cacans Warm. & 1 & $\mathrm{R}$ & Med & Ent & Zoo & NI & Fle \\
\hline \multirow{2}{*}{ APOCYNACEAE } & Nerium oleander L. & 3 & $\mathrm{~N}$ & Sma & Ent & Ane & Eve & Dry \\
\hline & Plumeria pudica Jacq. & 2 & $\mathrm{E}$ & Sma & NI & Ane & Eve & Dry \\
\hline \multirow{4}{*}{ ARALIACEAE } & Aralia rex (Ekman) J.Wen & 4 & E & NI & NI & Zoo & NI & Fle \\
\hline & Dendropanax cuneatus (DC.) Decne. \& Planch. & 1 & $\mathrm{R}$ & Sma & Ent & Zoo & Eve & Fle \\
\hline & Schefflera actinophylla (Endl.) Harms & 2 & $\mathrm{~N}$ & Sma & Ent & Zoo & Eve & Fle \\
\hline & Schefflera arboricola (Hayata) Merr. & 2 & $\mathrm{E}$ & Sma & Ent & Zoo & Eve & Fle \\
\hline ARAUCARIACEAE & Araucaria angustifolia (Bertol.) Kuntze & 11 & $\mathrm{~N}$ & Tall & Ane & Zoo & Eve & Dry \\
\hline \multirow{11}{*}{ ARECACEAE } & Archontophoenix cunninghamii $\mathrm{H}$. Wendl. \& Drude & 7 & $\mathrm{E}$ & Med & Ent & Zoo & Eve & Fle \\
\hline & Caryota mitis Lour. & 11 & $\mathrm{E}$ & Med & NI & Zoo & Eve & Fle \\
\hline & Cocos nucifera $\mathrm{L}$. & 2 & $\mathrm{~N}$ & Med & Ent & Hyd & Eve & Dry \\
\hline & Dypsis decaryi (Jum.) Beentje \& J.Dransf. & 2 & $\mathrm{E}$ & Sma & Ent & Zoo & Eve & Fle \\
\hline & Dypsis lutescens (H.Wendl.) Beentje \& J.Dransf. & 10 & E & Sma & Ent & Zoo & Eve & Fle \\
\hline & Euterpe edulis Mart. & 2 & $\mathrm{R}$ & Sma & Ent & Zoo & Eve & Fle \\
\hline & Geonoma schottiana Mart. & 1 & $\mathrm{R}$ & Sma & Ent & Zoo & Eve & Fle \\
\hline & Livistona chinensis (Jacq.) R.Br. ex Mart. & 21 & $\mathrm{E}$ & Med & Ent & Zoo & Eve & Fle \\
\hline & Phoenix roebelenii O’Brien & 16 & E & Sma & NI & Zoo & Eve & Fle \\
\hline & Roystonea borinquena O.F Cook & 27 & $\mathrm{E}$ & Sma & NI & Zoo & Eve & Fle \\
\hline & Syagrus romanzoffiana (Cham.) Glassman & 122 & $\mathrm{R}$ & Sma & Ent & Zoo & Eve & Fle \\
\hline ASPARAGACEAE & Yucca gigantea Lem. & 9 & $\mathrm{E}$ & Sma & Ent & Zoo & Eve & Fle \\
\hline ASTERACEAE & Baccharis dracunculifolia DC. & 1 & $\mathrm{R}$ & Sma & Ent & Ane & Eve & Dry \\
\hline \multirow{8}{*}{ BIGNONIACEAE } & Handroanthus chrysotrichus (Mart. ex DC.) Mattos & 10 & $\mathrm{~N}$ & Sma & Ent & Ane & Dec & Dry \\
\hline & $\begin{array}{l}\text { Handroanthus impetiginosus (Martius ex. DC.) } \\
\text { Mattos }\end{array}$ & 72 & $\mathrm{~N}$ & Sma & Ent & Ane & Dec & Dry \\
\hline & Handroanthus serratifolius (Vahl) S.Grose & 6 & $\mathrm{R}$ & Med & Ent & Ane & Dec & Dry \\
\hline & Handroanthus sp. & 2 & NI & Sma & Ent & Ane & Dec & Dry \\
\hline & Jacaranda mimosifolia D.Don & 1 & $\mathrm{E}$ & Med & Ent & Ane & Dec & Dry \\
\hline & Spathodea campanulata P.Beauv. & 6 & $\mathrm{E}$ & Med & Orn & Ane & Eve & Dry \\
\hline & Tabebuia roseoalba (Ridl.) Sandwith & 2 & $\mathrm{R}$ & Sma & Ent & Ane & Dec & Dry \\
\hline & Tecoma stans (L.) Juss. ex Kunth & 3 & $\mathrm{~N}$ & Sma & Ent & Ane & Eve & Dry \\
\hline
\end{tabular}

Sma: small-sized; Med: medium-sized; Ent: entomo-; Ane: anemo-; Aut: auto-; Chi: chiroptero-; Dec: deciduous; Dis: dispersal mode; Eve: evergreen; E: exotic; Fle: fleshy fruits; Fru: fruit type; Hyd: hydro-; N: native; NI: No Information; Ori: geographical origin; Orn: ornito-; Pol: pollination mode; R: regional. 
Table 1. Continued...

\begin{tabular}{|c|c|c|c|c|c|c|c|c|}
\hline Family & Species & Total & Ori & Size & Pol & Dis & Leaf & Fru \\
\hline \multirow{2}{*}{ BORAGINACEAE } & Cordia africana Lam. & 2 & $\mathrm{E}$ & Sma & Ent & Zoo & Eve & Fle \\
\hline & Cordia superba Cham. & 1 & $\mathrm{~N}$ & Sma & Ent & Zoo & Eve & Fle \\
\hline CASUARINACEAE & Casuarina equisetifolia L. & 1 & $\mathrm{E}$ & Tall & Ent & Zoo & Eve & Dry \\
\hline CHRYSOBALANACEAE & Licania tomentosa (Benth.) Fritsch & 4 & $\mathrm{~N}$ & Sma & Ane & Zoo & Eve & Fle \\
\hline COMBRETACEAE & Terminalia catappa L. & 3 & $\mathrm{~N}$ & Tall & Ane & Zoo & Dec & Dry \\
\hline \multirow{6}{*}{ CUPRESSACEAE } & Callitris preissii Miq. & 2 & $\mathrm{E}$ & Med & Orn & $\mathrm{NI}$ & Eve & Dry \\
\hline & Cupressus funebris Endl. & 3 & $\mathrm{E}$ & Tall & Ane & Ane & Eve & Dry \\
\hline & Cupressus lusitanica Mill. & 52 & $\mathrm{E}$ & Tall & Orn & Ane & Eve & Dry \\
\hline & Cupressus sempervirens $L$. & 14 & $\mathrm{E}$ & Tall & Orn & Ane & Eve & Dry \\
\hline & Thuja occidentalis L. & 1 & $\mathrm{E}$ & Med & Ane & Ane & Eve & Dry \\
\hline & Thuja sp. & 3 & $\mathrm{E}$ & Med & Ane & Ane & Eve & Dry \\
\hline \multirow{6}{*}{ EUPHORBIACEAE } & Actinostemon klotzschii (Didr.) Pax & 1 & $\mathrm{~N}$ & Sma & Ent & Zoo & Eve & Dry \\
\hline & Alchornea glandulosa Poepp. \& Endl. & 1 & $\mathrm{R}$ & Med & Ane & Zoo & Eve & Fle \\
\hline & Codiaeum variegatum (L.) Rumph. Ex A.Juss. & 6 & $\mathrm{E}$ & Sma & NI & NI & Eve & Dry \\
\hline & Euphorbiasp. & 1 & NI & Sma & Orn & Zoo & Eve & Dry \\
\hline & Jatropha curcas L. & 2 & $\mathrm{~N}$ & Sma & Ent & Aut & Dec & Dry \\
\hline & Sapium glandulosum (L.) Morong & 1 & $\mathrm{~N}$ & Med & Ent & Aut & Dec & Dry \\
\hline \multirow{19}{*}{ FABACEAE } & Acacia seyal Delile & 1 & $\mathrm{E}$ & Sma & Ent & Aut & Dec & Dry \\
\hline & Bauhinia variegata $\mathrm{L}$. & 9 & $\mathrm{E}$ & Sma & Ent & Aut & Dec & Dry \\
\hline & Caesalpinia pulcherrima (L.) Sw. & 2 & $\mathrm{~N}$ & Sma & Ent & Aut & Eve & Dry \\
\hline & Calliandra tweedii Benth. & 1 & $\mathrm{R}$ & Sma & Ent & Ane & Eve & Dry \\
\hline & Cassia fistula L. & 2 & $\mathrm{E}$ & Med & Ent & Aut & Dec & Dry \\
\hline & Dalbergia nigra (Vell.) Allemão ex Benth. & 2 & $\mathrm{R}$ & Med & Ent & Ane & Dec & Dry \\
\hline & Delonix regia (Bojer ex Hook.) Raf. & 8 & $\mathrm{E}$ & Sma & Ent & Aut & Dec & Dry \\
\hline & Enterolobium contortisiliquum (Vell.) Morong & 2 & $\mathrm{R}$ & Tall & Ent & Aut & Dec & Dry \\
\hline & Erythrina falcata Benth. & 1 & $\mathrm{R}$ & Med & Orn & Ane & Dec & Dry \\
\hline & Hymenaea courbaril L. & 1 & $\mathrm{R}$ & Med & Chi & Zoo & Eve & Dry \\
\hline & Leucaena leucocephala (Lam.) de Wit & 2 & $\mathrm{~N}$ & Sma & Ent & Aut & Eve & Dry \\
\hline & Lonchocarpus sericeus (Poir.) Kunth ex DC. & 1 & $\mathrm{~N}$ & Med & Ent & Zoo & Dec & Dry \\
\hline & Mimosa caesalpiniifolia Benth. & 8 & $\mathrm{~N}$ & Med & Ent & Aut & Dec & Dry \\
\hline & $\begin{array}{l}\text { Paubrasilia echinata (Lam.) Gagnon, H.C.Lima } \\
\text { \& G.P.Lewis }\end{array}$ & 4 & $\mathrm{R}$ & Sma & Ent & Ane & Eve & Dry \\
\hline & Peltophorum dubium (Spreng.) Taub. & 3 & $\mathrm{R}$ & Sma & Ent & Ane & Eve & Dry \\
\hline & $\begin{array}{l}\text { Poincianella pluviosa var. Peltophoroides (Benth.) } \\
\text { L.P. Queiroz }\end{array}$ & 237 & $\mathrm{R}$ & Med & Ent & Aut & Eve & Dry \\
\hline & Schizolobium parahyba (Vell.) Blake & 4 & $\mathrm{R}$ & Tall & Ent & Ane & Dec & Dry \\
\hline & $\begin{array}{l}\text { Senna macranthera (DC. ex Collad.) H.S.Irwin } \\
\text { \& Barneby }\end{array}$ & 1 & $\mathrm{R}$ & Sma & Ent & Aut & Dec & Dry \\
\hline & Tipuana tipu (Benth.) Kuntze & 1 & $\mathrm{E}$ & Med & Ent & Ane & Dec & Dry \\
\hline \multirow{2}{*}{ LAMIACEAE } & Callicarpa nudiflora Hook. \& Arn. & 1 & $\mathrm{E}$ & Sma & Ent & Zoo & Eve & Fle \\
\hline & Tectona grandis L.f. & 1 & $\mathrm{E}$ & Tall & Ent & Zoo & Dec & Fle \\
\hline LAURACEAE & Persea americana Mill. & 3 & $\mathrm{E}$ & Med & Ent & Zoo & Eve & Fle \\
\hline LECYTHIDACEAE & Couroupita guianensis Aubl. & 6 & $\mathrm{~N}$ & Med & Ent & Zoo & Dec & Fle \\
\hline \multirow{2}{*}{ LYTHRACEAE } & Lagerstroemia indica $\mathrm{L}$. & 2 & $\mathrm{E}$ & Sma & Ane & Aut & Eve & Dry \\
\hline & Punica granatum L. & 2 & $\mathrm{E}$ & Sma & Ent & Aut & Eve & Dry \\
\hline MALPIGHIACEAE & Malpighia emarginata DC. & 1 & $\mathrm{E}$ & Sma & Ent & Zoo & Eve & Fle \\
\hline
\end{tabular}

Sma: small-sized; Med: medium-sized; Ent: entomo-; Ane: anemo-; Aut: auto-; Chi: chiroptero-; Dec: deciduous; Dis: dispersal mode; Eve: evergreen; E: exotic; Fle: fleshy fruits; Fru: fruit type; Hyd: hydro-; N: native; NI: No Information; Ori: geographical origin; Orn: ornito-; Pol: pollination mode; R: regional. 
Table 1. Continued...

Family

MALVACEAE
MELASTOMATACEAE
MELIACEAE

MORACEAE

\begin{tabular}{l} 
MYRTACEAE \\
\hline OLEACEAE \\
\hline OXALIDACEAE \\
\hline PANDANACEAE \\
\hline PINACEAE \\
\hline PLATANACEAE \\
\hline POLYGONACEAE \\
\hline PRIMULACEAE \\
\hline RHAMNACEAE \\
\hline ROSACEAE \\
\hline RUBIACEAE \\
\hline
\end{tabular}

RUTACEAE
Species

Ceiba speciosa (A.St.-Hil.) Ravenna

Dombeya wallichii (Lindl.) Baill.

Guazuma ulmifolia Lam.

Hibiscus rosa-sinensis $\mathrm{L}$.

Luehea divaricata Mart. \& Zucc.

Pachira aquatica Aubl.

Pachira glabra Pasq.

\section{SALICACEAE}

\section{SAPINDACEAE}

SAPOTACEAE

Pleroma granulosum (Desr.) D. Don

Cedrela fissilis Vell.

Ficus benjamina $\mathrm{L}$.

Ficus microcarpa L.f.

Morus nigra L.

Calyptranthes brasiliensis Spreng.

Eucalyptus grandis W. Hill

Eugenia florida DC.

Eugenia involucrata DC.

Eugenia uniflora L.

Melaleuca leucadendra (L.) L.

Plinia cauliflora (Mart.) Kausel

Psidium guajava L.

Siphoneugena densiflora O.Berg

Syzygium cumini (L.) Skeels

Ligustrum lucidum W.T.Aiton

Averrhoa carambola L.

Pandanus tectorius Parkinson ex Du Roi.

Pinus patula Schiede ex Schltdl. \& Cham.

Platanus $x$ acerifolia (Ait.) Willd

Triplaris americana L.

Triplaris caracasana Cham.

Hovenia dulcis Thunb.

Genipa americana L.

Citrus sp.

Citrus $x$ limon (L.) Osbeck Niederl.

Total Ori Size Pol Dis Leaf Fru

$\mathrm{R}$ Med Chi Ane Dec Dry

Callistemon viminalis (Sol. ex Gaertn.) G.Don

2 E Sma Ent Zoo Eve Dry

R Sma Ent Zoo Eve Dry

$\begin{array}{llllll}1 & \text { R } & \text { Sma } & \text { Ent Zoo } & \text { Eve } & \text { Dry } \\ 4 & \text { E } & \text { Sma } & \text { Orn NI Eve Dry }\end{array}$

$1 \mathrm{R}$ Med Ent Ane Dec Dry

1 N Sma Chi Aut Eve Dry

4 R Sma Chi Aut Eve Dry

$55 \mathrm{~N}$ Sma Ent Ane Eve Dry

$1 \quad \mathrm{R}$ Med Ent Ane Dec Dry

$59 \mathrm{~N}$ Med Ent Zoo Eve Fle

2 E Med Ent Zoo Eve Fle

6 E Sma Ent Zoo Dec Fle

13 E Sma Orn Ane Eve Dry

Myrsine guianensis (Aubl.) Kuntze

Eriobotrya japonica (Thunb.) Lindl. $\quad 1 \quad \mathrm{~N}$ Sma Ent Zoo Eve Fle

1 R Sma Ent Zoo NI Fle

1 E Tall Ent Ane Eve Fle

1 R Sma Ent Zoo Eve Fle

1 R Sma Ent Zoo Dec Fle

21 R $\quad$ Sma Ent Zoo Eve Fle

4 E Sma Ent Ane Eve Dry

$11 \mathrm{R}$ Med Ent Zoo Eve Fle

$26 \mathrm{~N}$ Sma Ent Zoo Eve Fle

2 R Sma Ent Zoo Eve Fle

$23 \mathrm{~N}$ Med Ent Zoo Eve Fle

3 E Sma Ent Zoo Eve Dry

1 E Sma Ent Zoo Eve Fle

1 E Sma Ent Zoo Dec Fle

1 E Tall Ane Ane Eve Dry

1 E Tall Ane Ane Dec Dry

$1 \mathrm{~N}$ Med Ent Ane Eve Dry

2 E Sma Ent Ane Dec Dry

$\begin{array}{lllllll}1 & \mathrm{R} & \text { Med } & \text { Ent } & \text { Zoo } & \text { Eve } & \text { Fle } \\ 1 & \mathrm{R} & \text { Med } & \text { Ent } & \text { Zoo } & \text { Dec } & \text { Dry }\end{array}$

1 NI Sma Ent Zoo Eve Fle

$4 \mathrm{~N}$ Sma Ent Zoo Eve Fle

Murraya paniculata (L.) Jack $\quad 5 \quad$ E Sma Ent Zoo Eve Fle

Casearia sylvestris Sw. $\quad 1 \quad$ R $\quad$ Sma Ent Zoo Eve Dry

Allophylus edulis (A.St.-Hil. et al.) Hieron. ex $\quad 6 \quad$ R Sma Ent Zoo Dec Fle

Micropholis guyanensis (A.DC.) Pierre $\quad 1 \quad$ R Tall Ent Zoo NI Fle Brunfelsia uniflora (Pohl) D.Don $\quad 1 \quad$ R Sma Ent Ane Eve Dry VERBENACEAE Duranta erecta L.

$30 \quad \mathrm{R}$ Sma Orn Ane Eve Dry

Sma: small-sized; Med: medium-sized; Ent: entomo-; Ane: anemo-; Aut: auto-; Chi: chiroptero-; Dec: deciduous; Dis: dispersal mode; Eve: evergreen; E: exotic; Fle: fleshy fruits; Fru: fruit type; Hyd: hydro-; N: native; NI: No Information; Ori: geographical origin; Orn: ornito-; Pol: pollination mode; R: regional.

abundance pattern there were $25 \%$ exotic individuals, $31 \%$ native, and $43 \%$ regional. The exotic group was more equitable than the native and regional groups (Table 2).
The two dominant species (Poincianella pluviosa and Syagrus romanzoffiana) are regional and accounted for $32 \%$ of the total abundance. There was a notable presence of few abundant species, $72 \%$ of the total 
species showed equal or less than five individuals. Despite the two dominant species, the regional group was also the group with the highest percentage of rare species (Figure 2).

The diversity indices (HF') were considered low mainly for HF'ind, and showed great variations in the different categories, ranging from 0.42 (leaf life span) to 1.09 (dispersal mode). The exotic species showed higher $\mathrm{H}$-values in relation to the native and regional species in a majority of the categories (Table 3).

Most species were classified as: a) small-sized (60\% of the total species) $(\mathrm{F}=141.4, \mathrm{gl}=2, \mathrm{p}<0.001)$; b) entomophilous ( $80 \%)(\mathrm{F}=16.6, \mathrm{gl}=3, \mathrm{p}<0.001)$; c) zoochoric $(57 \%)(\mathrm{F}=21.0, \mathrm{gl}=3, \mathrm{p}<0.001)$; d) evergreen $(71 \%)(\mathrm{t}=-9.8, \mathrm{gl}=10, \mathrm{p}<0.05)$; and e) with dry fruits $(56 \%)(\mathrm{t}=-3.2, \mathrm{gl}=10, \mathrm{p}<0.05)$. The general pattern for individuals was more heterogeneous, with a predominance of the following attributes: a) small (48\%) and medium-sized (41\%) ( $\mathrm{F}=7.5, \mathrm{gl}=2, \mathrm{p}<0.01) ; \mathrm{b})$ entomophilous (84\%) $(\mathrm{F}=19.2, \mathrm{gl}=3, \mathrm{p}<0.001) ; \mathrm{c})$ zoochoric $(42 \%)$, anemochoric (29\%), and autochoric (28\%) $(\mathrm{F}=14.0$, $\mathrm{gl}=3, \mathrm{p}<0.001) ; \mathrm{d})$ evergreen (83\%) $(\mathrm{t}=-5.3, \mathrm{gl}=10$, $\mathrm{p}<0.05)$; and (e) with dry fruits $(65 \%)(\mathrm{t}=1.9, \mathrm{gl}=10$, $\mathrm{p}<0.05$ ) (Figure 3).

Table 2. Parameters of the plant assemblages in eight green public spaces in Alfenas, Minas Gerais State, Brazil.

\begin{tabular}{lccc}
\multicolumn{1}{c}{ Origin } & Abundance & Richness & $\begin{array}{c}\text { Evenness } \\
\left(\mathbf{J}^{\prime}\right)\end{array}$ \\
\hline Regional & 485 & 40 & 0.37 \\
Native & 359 & 28 & 0.51 \\
\hline Exotic & 290 & 48 & 0.68 \\
\hline No Information & 4 & 3 & - \\
\hline Total & 1138 & 119 & 0.72 \\
\hline
\end{tabular}

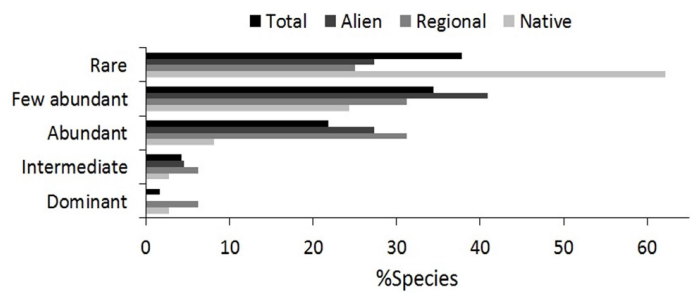

Figure 2. Percentages of species by abundance class. Rare: species with only one individual; Few abundant: 2-5individuals; Abundant: 6-50 individuals; Intermediate: 51-100 individuals; Dominant: > 100 individuals.
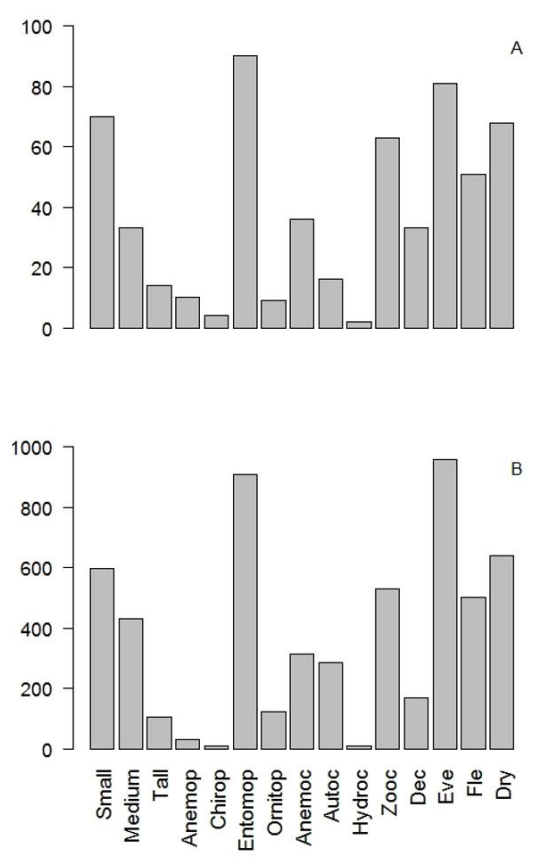

Figure 3. Number of species (A) (total richness: 119) and individuals (B) (total abundance: 1138) from urban plant assemblages in terms of their functional attributes. Small: Small-sized; Medium: Medium-sized; Tall: Tall-sized; Anemop: Anemophily; Chirop: Chiropterophily; Entomop: Entomophily; Ornitop: Ornithophily; Anemoc: Anemochory; Autoc: Autochory; Hydroc: Hydrochory; Zooc: Zoochory; Dec: Deciduous leaves; Eve: Evergreen leaves; Fle: Fleshy fruits; Dry: Dry fruits.

Table 3. Functional Diversity Index ${ }^{\star}\left(\mathrm{HF}^{\prime}\right)$, calculated based on the proportion of individuals (HF'ind) and species (HF'sp) in each functional group of the urban flora.

\begin{tabular}{|c|c|c|c|c|c|c|c|c|c|c|}
\hline & \multicolumn{5}{|c|}{ HF'ind } & \multicolumn{5}{|c|}{ HF'sp } \\
\hline & Size & Pollination & Dispersal & Leaves & Fruit & Size & Pollination & Dispersal & Leaves & Fruit \\
\hline Regional & 0.75 & 0.34 & 0.97 & 0.24 & 0.65 & 0.27 & 0.25 & 0.28 & 0.26 & 0.26 \\
\hline Natives & 0.77 & 0.38 & 0.86 & 0.67 & 0.73 & 0.21 & 0.19 & 0.23 & 0.19 & 0.20 \\
\hline Exotic & 0.92 & 0.75 & 0.84 & 0.53 & 0.72 & 0.32 & 0.28 & 0.31 & 0.27 & 0.30 \\
\hline Total & 0.93 & 0.53 & 1.09 & 0.42 & 0.70 & 0.92 & 0.71 & 1.04 & 0.6 & 0.68 \\
\hline
\end{tabular}

*Adapted from Shannon's Diversity Index (Shannon \& Weaver, 1948). 


\section{DISCUSSION}

\subsection{Species diversity}

Intensively managed urban ecosystems are not necessarily barriers to biodiversity. Our results suggest that those spaces can act as refuges for rich plant communities. The species richness in the observed green areas was considered high in comparison with other studies undertaken in Brazil (Almeida \& Barbosa (2010) reported 45 species; Kramer \& Krupek (2012) 98 species; and Raber \& Rebelato (2010) 45 species). However, small patches of natural remnant vegetation in the study region often harbor richer communities (Carneiro et al., 2016; Nunes et al., 2003). It suggests the need to consider urban spaces as complementary areas for the conservation of natural forest fragments. The species compositions of the urban green areas also were quite distinct from natural vegetation, as expected, with the marked presence of exotic species. The same pattern has been reported in other cities (Almeida \& Barbosa, 2010; Cardoso-Leite et al., 2014; Santos et al., 2012; Wang et al., 2012). Despite increasing species richness at a local level, the introduction of exotic species to urban afforestation sites should be viewed with caution. The impact of exotic plants is an ongoing issue and numerous undesirable consequences have been reported. The consequences include competition with native species and their subsequent population declines (Vidra et al., 2007), the homogenization of the compositions of urban floras (McKinney, 2006), damage to the associated local fauna (Corbet et al., 2001), and biological invasions (Shackleton \& Shackleton, 2016).

The persistence of the native flora is not always feasible in cities due to the peculiarities of urban environments and their highly restrictive conditions (Sukopp, 2004; Knapp et al., 2010). High levels of disturbance, reduced sizes of vegetation refuges, and little or no connectivity between them constitute unfavorable conditions that can hinder many complex biological processes and limit the presence of rare native specialist species in cities (Ordóñez \& Duinker, 2012; Qing et al., 2015). Therefore it would be desirable for urban afforestation projects to incorporate mechanisms that would stimulate ecological complexity, for example, by increasing size and connectivity among habitat patches (Mörtberg, 2001).
Another relevant point about urban plant communities is their low species evenness. The greater part of the vegetation cover in cities usually consists of only one or a few dominant species, with many other less abundant taxa (Pauleit et al., 2002; Veloso et al., 2014). The two dominant species in Alfenas area accounted for more than $30 \%$ of the total abundance, and some of the common species in our study are found in other cities throughout Brazil (Kramer \& Krupek, 2012; Santos et al., 2012; Freitas et al., 2015). In general, more diverse areas tend to be ecologically more robust than sites dominated by just a small set of species (Nagendra \& Gopal, 2010), and it has been recommended that a single species not exceed $15 \%$ of the total number of planted trees (Redin et al., 2010).

\subsection{Functional diversity}

Functional diversity should be considered as a conservation tool in urban tree projects, as the diversity of ecosystem services performed far more important than the simple numbers of species (Flynn et al., 2009). Human choices regarding which species will form urban plant communities will act as strong selective filters of the richness and functional plant types found in urban habitats (Williams et al., 2008; Knapp et al., 2010). As such, the floristic compositions of the persistent floras of those areas typically combine both cultivated and ornamental species, regardless of their functional or phylogenetic diversity. The functional diversity index in the present study site was considered low for all ecological attributes. The lack of literature investigating that aspect in other Brazilian cities makes comparisons difficult. However, other works have pointed out that increases in tree richness do not necessarily guarantee increased functional diversity, with some ecological redundancy in urban green spaces (Knapp et al., 2008; Dolan et al., 2017). We encourage other researchers to adopt that same perspective in tropical regions.

The patterns of ecological traits found here revealed some similarities with those seen in Semideciduous Seasonal forest fragments, especially in relation to dispersal and pollination syndromes, with most species being zoochoric and entomophilous (Kinoshita et al., 2006; Vale et al., 2011). The presence of species with functional characteristics equivalent to the native flora is desirable and can transform cities into permanent ecological corridors - connecting nearby forest 
fragments and increasing the permeability of the urban matrix (Munshi-South, 2012). Additionally, the urban flora itself will benefit from those interactions, as the ecological processes essential to their reproduction and persistence will be maintained (Corlett, 2005).

Regarding tree size and fruit type, altered environments commonly favor plants with ruderal life history, smaller size, and dry fruits (Knapp et al., 2012; Williams et al., 2008). The establishment of other functional traits can also be restricted by intentional choices of the species to be cultivated - benefiting plants with characteristics compatible with the management of cultivated spaces. Small trees, for example, may be more suitable for sidewalks and urban power lines (Guimarães, 2006), while species with dry fruits tend to be favored over "messy" fleshy fruits. However, disregarding the ecological aspects of the urban flora can have negative effects on biodiversity (Cunha et al., 2006). The absence of larger trees can reduce the amounts and varieties of resources available to local wildlife (Guimarães, 2006), besides decreasing human thermal comfort (Araujo et al., 2017). Similarly, the lack of zoochoric species can impact local fauna that depends on fruits as food resources. Foraging on urban fruit trees has been observed in many different animals groups, such as bats (Corlett, 2005), monkeys (Cunha et al., 2006), lizards (González-García et al., 2009), and birds (Pauw \& Louw, 2012).

\section{CONCLUSIONS}

The study of plant diversity represents a promising tool for the conservation of biodiversity in urban ecosystems. Since those habitats are becoming increasingly common with the expansion of human populations, the determination of taxonomic and functional patterns will be critical to understand community dynamics and for subsidizing management and conservation strategies. Our results indicate that urban green areas can function as refuges for rich plant communities with significant biodiversity conservation potential, although they are currently poor in regional native species and functional diversity. Urban green areas should therefore best be viewed as complementary strategies to aid in preserving forest remnants, since their floristic and functional compositions greatly differ. Ideally, the choice of species for urban floras should take into account not only socio-economic benefits to human populations, but also natural aspects that can contribute to the ecological integrity and complexity of those environments. We encourage continuing investigations of functional patterns as a tool for understanding the dynamics of biological communities in urban ecosystems. Furthermore, large regional native trees and fleshy fruits should be included in suitable areas to increase the environmental balance, maintain and attract the regional native frugivorous fauna.

\section{ACKNOWLEDGEMENTS}

We thank Almeida, T.H.M.P. for help in work field, Sampaio, M.B. and anonymous reviewers for their constructive comments, and FAPEMIG for granting a scholarship to one of the authors.

\section{SUBMISSION STATUS}

Received: 1 nov., 2017

Accepted: 30 jun., 2018

\section{CORRESPONDENCE TO}

\section{Flavio Ramos}

Laboratório de Ecologia de Fragmentos Florestais, Programa de Pós-graduação em Ciências Ambientais, Instituto de Ciências da Natureza, Universidade Federal de Alfenas UNIFAL, Rua Gabriel Monteiro da Silva, 700, CEP 37130-000, Centro, Alfenas, MG, Brasil e-mail: fnramos@gmail.com

\section{FINANCIAL SUPPORT}

Fundação de Amparo à Pesquisa do Estado de Minas Gerais - FAPEMIG (Grant/Award Number: 7716/2013).

\section{REFERENCES}

Almeida JR, Barbosa CG. Diagnóstico da arborização urbana da cidade de Cacoal - RO. Revista da Sociedade Brasileira de Arborização Urbana 2010; 5(1): 61-81. http:// dx.doi.org/10.5380/revsbau.v5i1.66239.

Alvares CC, Stape JL, Sentelhas PC, Golçalves JLM, Sparovek G. Köppen's climate classification map for Brazil. Meteorologische Zeitschrift (Berlin) 2014; 22(6): 711-728. http://dx.doi.org/10.1127/0941-2948/2013/0507. 
Araujo AM, Saraiva ALBC, Grígio AM. Conforto térmico humano: um estudo de caso em três praças públicas do bairro Centro, Mossoró (RN). Revista Geointerações 2017; 1(2): 31-50.

Aronson MFJ, Handel SN, Clemants SE. Fruit type, life form and origin determine the success of woody plant invaders in an urban landscape. Biological Invasions 2007; 9(4): 465-475. http://dx.doi.org/10.1007/s10530-006-9053-1.

Bigirimana J, Bogaert J, Canniere C, Lejoly J, Parmentier I. Alien plant species dominate the vegetation in a city of Sub-Saharan Africa. Landscape and Urban Planning 2011; 100(3): 251-267. http://dx.doi.org/10.1016/j. landurbplan.2010.12.012.

Cardoso-Leite E, Faria LC, Capelo FFM, Tonello KC, Castello ACD. Floristic composition of urban afforestation in Sorocaba, São Paulo state, Brazil. Revista da Sociedade Brasileira de Arborização Urbana 2014; 9(1): 125-141.

Carneiro MS, Campos CCF, Ramos FN, Santos FAM. Spatial species turnover maintains high diversities in a tree assemblage of a fragmented tropical landscape. Ecosphere 2016; 7(10): 1-11. http://dx.doi.org/10.1002/ecs2.1500.

Corbet SA, Bee J, Dasmahapatra K, Gale S, Gorringe E, La Ferla B et al. Native or exotic? Double or single? Evaluating plants for pollinator-friendly gardens. Annals of Botany 2001; 87(2): 219-232. http://dx.doi.org/10.1006/ anbo.2000.1322.

Corlett RT. Interactions between birds, fruit bats and exotic plants in urban Hong Kong, South China. Urban Ecosystems 2005; 8(3-4): 275-283. http://dx.doi.org/10.1007/ s11252-005-3260-x.

Cornelissen JHC, Lavorel S, Garnier E, Díaz S, Buchmann $\mathrm{N}$, Gurvich DE et al. A handbook of protocols for standardised and easy measurement of plant functional traits worldwide. Australian Journal of Botany 2003; 51(4): 335-380. http://dx.doi.org/10.1071/BT02124.

Cunha AA, Vieira MV, Grelle CEV. Preliminary observations on habitat, support use and diet in two nonnative primates in an urban Atlantic forest fragment: the capuchin monkey (Cebus sp.) and the common marmoset (Callithrix jacchus) in the Tijuca forest, Rio de Janeiro. Urban Ecosystems 2006; 9(4): 351-359. http://dx.doi. org/10.1007/s11252-006-0005-4.

Díaz S, Cabido M. Vive la différence: plant functional diversity matters to ecosystem processes. Trends in Ecology \& Evolution 2001; 16(11): 646-655. http://dx.doi. org/10.1016/S0169-5347(01)02283-2.

Dolan RW, Aronson MFJ, Hipp AL. Floristic response to urbanization: Filtering of the bioregional flora in Indianapolis, Indiana, USA. American Journal of Botany 2017; 104(8): 1179-1187. http://dx.doi.org/10.3732/ ajb.1700136. PMid:28794058.

Duncan RP, Clemants SE, Corlett RT, Hahs AK, McCarthy MA, McDonnell MJ et al. Plants traits and extinction in urban areas: a meta-analysis of 11 cities. Global Ecology and Biogeography 2011; 20(4): 509-519. http://dx.doi. org/10.1111/j.1466-8238.2010.00633.x.

Flynn DFB, Gogol-Prokurat M, Nogeire T, Molinari N, Richers BT, Lin BB et al. Loss of functional diversity under land use intensification across multiple taxa. Ecology Letters 2009; 12(1): 22-33. http://dx.doi.org/10.1111/j.14610248.2008.01255.x. PMid:19087109.

Freitas WK, Pinheiro MAS, Abrahão LLF. Análise da arborização de quatro praças no bairro da Tijuca, RJ, Brasil. Floresta e Ambiente 2015; 22(1): 23-31. http:// dx.doi.org/10.1590/2179-8087.025612.

González-García A, Belliure J, Gómez-Sal A, Dávila P. The role of urban greenspaces in fauna conservation: the case of the iguana Ctenosaura similis in the "patios" of León city, Nicaragua. Biodiversity and Conservation 2009; 18(7): 1909-1920. http://dx.doi.org/10.1007/s10531-008-9564-4.

Guimarães M. Há mais aves nos grandes centros urbanos hoje? Ciência \& Cultura. 2006; 58(2): 14-15.

Instituto Brasileiro de Geografia e Estatística - IBGE. Manuais técnicos em geociências: manual técnico da vegetação brasileira. Rio de Janeiro: IBGE; 2012.

Isernhagen I, Le Bourlegat JMG, Carboni M. Trazendo a riqueza arbórea regional para dentro das cidades: possibilidades, limitações e benefícios. Revista da Sociedade Brasileira de Arborização Urbana 2009; 4(2): 117-138. http://dx.doi.org/10.5380/revsbau.v4i2.66277.

Kinoshita LS, Torres RB, Forni-Martins ER, Spinelli T, Ahn YJ, Constâncio SS. Composicão florística e síndromes de polinização e dispersão da mata do Sítio São Francisco, Campinas, SP, Brasil. Acta Botanica Brasílica 2006; 20(2): 313-327. http://dx.doi.org/10.1590/S010233062006000200007 .

Knapp S, Dinsmore L, Fissore C, Hobbie SE, Jakobsdottir I, Kattge J et al. Phylogenetic and functional characteristics of household yard floras and their changes along an urbanization gradient. Ecology 2012; 93(8): S83-S98. http://dx.doi.org/10.1890/11-0392.1.

Knapp S, Kühn I, Schweiger O, Klotz S. Challenging urban species diversity: contrasting phylogenetic patterns across plant functional groups in Germany. Ecology Letters 2008; 11(10): 1054-1064. http://dx.doi.org/10.1111/j.14610248.2008.01217.x. PMid:18616547.

Knapp S, Kühn I, Stolle J, Klotz S. Changes in the functional composition of a Central European urban flora over three centuries. Perspectives in Plant Ecology, Evolution and Systematics 2010; 12(3): 235-244. http:// dx.doi.org/10.1016/j.ppees.2009.11.001.

Köppen W. Climatologia. 1st ed. Mexico City: Fondo Cultura Economica; 1948.

Kowarik I. Novel urban ecosystems, biodiversity, and conservation. Environmental Pollution 2011; 159(8-9): 
1974-1983. http://dx.doi.org/10.1016/j.envpol.2011.02.022. PMid:21435761.

Kramer JA, Krupek RA. Caracterização florística e ecológica da arborização de praças públicas do município de Guarapuava, PR. Revista Árvore 2012; 36(4): 647-658. http://dx.doi.org/10.1590/S0100-67622012000400007.

McKinney ML. Urbanization as a major cause of biotic homogenization. Biological Conservation 2006; 127(3): 247-260. http://dx.doi.org/10.1016/j.biocon.2005.09.005.

Mörtberg UM. Resident bird species in urban forest remnants; landscape and habitat perspectives. Landscape Ecology 2001; 16(3): 193-203. http://dx.doi.org/10.1023/A:1011190902041.

Munshi-South J. Urban landscape genetics: canopy cover predicts gene flow between white-footed mouse (Peromyscus leucopus) populations in New York City. Molecular Ecology 2012; 21(6): 1360-1378. http://dx.doi. org/10.1111/j.1365-294X.2012.05476.x. PMid:22320856.

Nagendra H, Gopal D. Street trees in Bangalore: Density, diversity, composition and distribution. Urban Forestry \& Urban Greening 2010; 9(2): 129-137. http://dx.doi. org/10.1016/j.ufug.2009.12.005.

Nunes YRF, Mendonça AVR, Botezelli L, Machado ELM, Oliveira-Filho AT. Variações da fisionomia, diversidade e composição de guildas da comunidade arbórea em um fragmento de floresta semidecidual em Lavras, MG. Acta Botanica Brasílica 2003; 17(2): 213-229. http://dx.doi. org/10.1590/S0102-33062003000200005.

Ordóñez C, Duinker PN. Ecological integrity in urban forests. Urban Ecosystems 2012; 15(4): 863-877. http:// dx.doi.org/10.1007/s11252-012-0235-6.

Pauleit S, Jones N, Garcia Martin G, Garcia Valdecantos JL, Riviere LM, Vidal Beaudet L et al. Tree establishment practice in towns and cities-results from a European survey. Urban Forestry \& Urban Greening 2002; 1(2): 83-96. http:// dx.doi.org/10.1078/1618-8667-00009.

Pauw A, Louw K. Urbanization drives a reduction in functional diversity in a guild of nectar-feeding birds. Ecology and Society 2012; 17(2): 27. http://dx.doi. org/10.5751/ES-04758-170227.

Qing X, Yutong Z, Shenggao L. Assessment of heavy metal pollution and human health risk in urban soils of steel industrial city (Anshan), Liaoning, Northeast China. Ecotoxicology and Environmental Safety 2015; 120: 377-385. http://dx.doi.org/10.1016/j.ecoenv.2015.06.019. PMid:26114257.

Raber AP, Rebelato GS. Arborização viária no município de Colorado, RS - Brasil: análise quali-quantitativa. Revista da Sociedade Brasileira de Arborização Urbana 2010; 5(1): 183-199. http://dx.doi.org/10.5380/revsbau.v5i1.66260.

Redin CG, Vogel C, Trojahn CDP, Gracioli CR, Longhi SJ. Análise da arborização urbana em cinco praças do município de Cachoeira do Sul, RS. Revista da Sociedade Brasileira de Arborização Urbana 2010; 5(3): 149-164.
Roy S, Byrne J, Pickering C. A systematic quantitative review of urban tree benefits, costs, and assessment methods across cities in different climatic zones. Urban Forestry \& Urban Greening 2012; 11(4): 351-363. http:// dx.doi.org/10.1016/j.ufug.2012.06.006.

Santos TOB, Lisboa CMCA, Carvalho FG. Análise da arborização viária do bairro de Petropólis, Natal, RN: uma abordagem para diagnóstico e planejamento da flora urbana. Revista da Sociedade Brasileira de Arborização Urbana 2012; 7(4): 90-106. http://dx.doi.org/10.5380/ revsbau.v7i4.66546.

Schlaepfer MA, Sax DF, Olden JD. The potential conservation value of non-native species. Conservation Biology 2011; 25(3): 428-437. http://dx.doi.org/10.1111/j.15231739.2010.01646.x. PMid:21342267.

Shackleton C, Shackleton RT. Knowledge, perceptions and willingness to control designated invasive tree species in urban household gardens in South Africa. Biological Invasions 2016; 18(6): 1599-1609. http://dx.doi.org/10.1007/ s10530-016-1104-7.

Shannon CE, Weaver W. The mathematical theory of communication. Illinois: University of Illinois Press; 1948.

Sukopp H. Human-caused impact on preserved vegetation. Landscape and Urban Planning 2004; 68(4): 347-355. http:// dx.doi.org/10.1016/S0169-2046(03)00152-X.

Töpfer K, Wolfensohn JD, Lash J. World resources 2000 2001. Amsterdam: Elsevier Science; 2000.

Vale VS, Dorneles MC, Schiavini I, Mendonça ET, Almeida CG, Silva PA et al. Grupos funcionais e sua importância ecológica na vegetação arbórea em um remanescente florestal urbano, Uberlândia, MG. Revista Natureza Online 2011; 9(2): 67-75.

Veloso MDM, Braga LL, Rodrigues PMS, Santos MR, Miranda WO, Brandao DO et al. Caracterização da arborização urbana em três ambientes na cidade de Montes Claros, MG. Revista da Sociedade Brasileira de Arborização Urbana 2014; 9(2): 118-133. http://dx.doi. org/10.5380/revsbau.v9i2.63209.

Vidra RL, Shear TH, Stucky JM. Effects of vegetation removal on native understory recovery in an exotic-rich urban forest. The Journal of the Torrey Botanical Society 2007; 134(3): 410-419. http://dx.doi.org/10.3159/10955674(2007)134[410:EOVRON]2.0.CO;2.

Wang H-F, MacGregor-Fors I, López-Pujol J. Warmtemperate, immense, and sprawling: plant diversity drivers in urban Beijing, China. Plant Ecology 2012; 213(6): 967-992. http://dx.doi.org/10.1007/s11258-012-0058-9.

Williams NSG, Schwartz MW, Vesk PA, McCarthy MA, Hahs AK, Clemants SE et al. A conceptual framework for predicting the effects of urban environments on floras. Journal of Ecology 2008; 97(1): 4-9. http://dx.doi. org/10.1111/j.1365-2745.2008.01460.x. 